\title{
Manegold de Lautenbach
}

\section{Martine Hiebel}

Traducteur : Martine Hiebel

\section{OpenEdition \\ Journals}

Édition électronique

URL : https://journals.openedition.org/rbnu/2738

DOI : $10.4000 /$ rbnu. 2738

ISSN : 2679-6104

\section{Éditeur}

Bibliothèque nationale et universitaire de Strasbourg

\section{Édition imprimée}

Date de publication : 1 novembre 2012

Pagination : $72-77$

ISSN : 2109-2761

\section{Référence électronique}

Martine Hiebel, « Manegold de Lautenbach », La Revue de la BNU [En ligne], 6 | 2012, mis en ligne le 01 novembre 2012, consulté le 08 août 2021. URL : http://journals.openedition.org/rbnu/2738; DOI : https://doi.org/10.4000/rbnu.2738

\section{(c) (i) (2) (2)}

La Revue de la BNU est mise à disposition selon les termes de la Licence Creative Commons Attribution - Pas d'Utilisation Commerciale - Partage dans les Mêmes Conditions 4.0 International. 


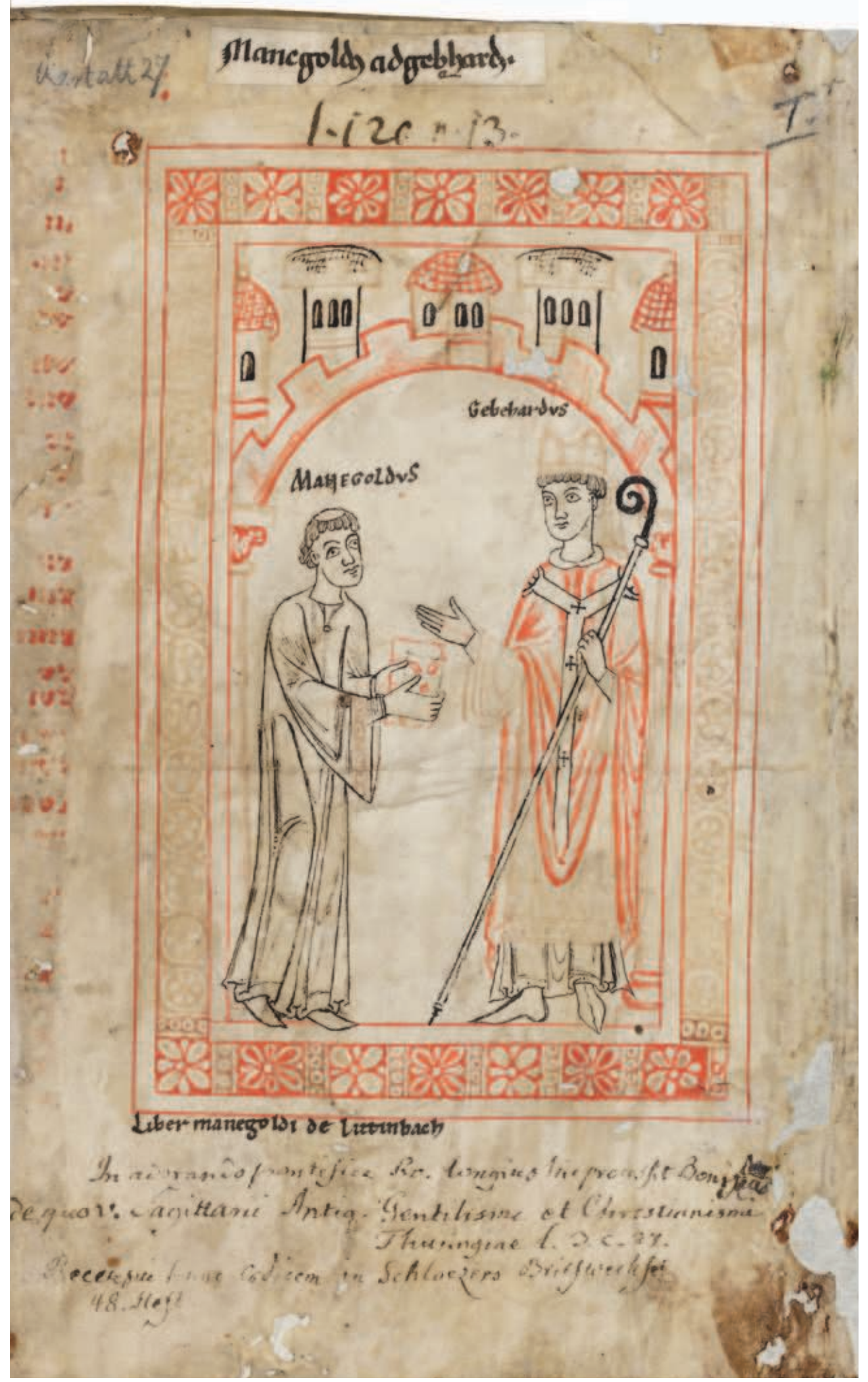

Illustration ouvrant le manuscrit du Livre à Gebhard,

appelé aussi " manuscrit de Rastatt "

(coll. Badische Landesbibliothek Karlsruhe) 


\section{Manegold de Lautenbach}

" En avant et du nouveau !", criait un certain Manegold dans le drame Abélard imaginé en 1845 par Charles de Rémusat ${ }^{1}$. La philosophie du haut Moyen Âge y semble convoquée, car Abélard eut pour maître Manegold de Lautenbach ; mais après avoir signé une brillante œuvre théorique et polémique, ce dernier nom sombra dans les oubliettes de l'Histoire ${ }^{2}$. Des textes de Manegold furent cependant établis, et mis en perspective en latin par Kuno Francke pour figurer parmi les Monumenta Germaniae Historica ${ }^{3}$, en 1891, même si l'on y déplorait la démesure et l'enflure du style de cet auteur. Une de ses œuvres principales, le Livre à Gebhard, est en tout cas restée jusqu'à nos jours inédite en français ; c'est un extrait de ce texte qui est proposé ici en traduction littérale.

Il faut reconnaître qu'autour de 1080 les circonstances prêtaient aux excès : le Saint Empire romain germanique auquel appartenait Lautenbach était secoué par la querelle des Investitures qui troublait toute la chrétienté. Dépourvu des institutions nécessaires, il ne pouvait maintenir sa puissance sur les princes qu'avec l'appui de l'Eglise. Or une réforme en cours visait à la dégager de la prérogative impériale dans ses nominations appelées investitures, donc dans son fonctionnement global, pour la rendre à sa vocation sacrée, spirituelle et morale. Hildebrand en était l'instigateur dès la première moitié du $11^{\mathrm{e}}$ siècle et, une fois élu au Saint-Siège sous le nom de Grégoire VII, la mena finalement à bien en dépit de l'empereur Henri IV : il fallut à ce pape surmonter sa propre déposition réitérée en passant par divers synodes, par l'abolition du serment prêté à l'empereur, par des excommunications de part et d'autre de Canossa ${ }^{4}$, par la nomination d'un antiroi qui déclencherait un antipape et par un embrasement général de lutte armée...
$\mathrm{Au}$ cours de cette période, sans doute parti de Lautenbach (dans l'actuel département du HautRhin) où il serait né vers 1030, Manegold devint rapidement à Paris et loin à la ronde " le maître des maîtres modernes "; il fit probablement école de manière gratuite, itinérante autant qu'exigeante, avec son propre foyer avant de se retirer dans le même village alsacien. Quand il y fut reçu par un chapitre de chanoines réguliers de saint Augustin, la querelle des Investitures le rattrapa, vraisemblablement dès qu'il jeta, peut-être diffusa, les premières idées du Livre à Gebhard. Sa rédaction se déroula sur ordre canonial, par étapes et malgré les obstacles. Dirigé contre le primat de la dialectique néo-platonicienne, le Livre contre Wolfhelm semble avoir eu le temps d'être achevé par Manegold ; mais, à cause de son auteur, le petit monastère de Lautenbach fut la proie des troupes impériales qui le détruisirent et le vidèrent de ses membres en 1080.

Le futur Livre à Gebhard poursuivit alors de plus belle, avec son auteur fugitif, sa route contre le parti de l'empereur : figurant parmi les textes complémentaires cités par R. Ziomkowski ${ }^{5}$, son prologue révèle de poignants détails sur la fuite, sur la mission nouvelle et sur la foi de Manegold à travers une dédicace aussi humble que grandiose, qui s'adresse à Gebhard, archevêque de Salzbourg, et que représente une enluminure singulièrement " collée ", comme le précise K. Francke, sur la couverture de cet ouvrage. L'exil entraînait Manegold à l'est de l'Alsace et dans une tout autre carrière. Car une lettre composée par le prélat " progrégorien " de Salzbourg - dont la citadelle censée prévenir les attaques du Saint Empire romain germanique se dresse depuis lors dans la cité mozartienne - fut mise en circulation en même temps qu'un 
pamphlet " antigrégorien " signé par Thierry, évêque de Verdun, sous la plume d'un Wenricus ou Werricus, écolâtre ${ }^{6}$ à Trèves : la publication quasi simultanée de ces deux écrits opposés parait avoir été l'étincelle d'où l'ouvrage de Manegold jaillit, avec une impertinente pertinence, au milieu des orages politico-religieux. Se tournant à la fois vers Gebhard de Salzbourg et contre les partisans de l'empereur, combinant sa formation poussée et ses convictions passionnées, il entendait contribuer à rendre l'Eglise digne du Christ.

Le brûlot que constitue ce livre interminable nous est d'ailleurs parvenu sous une forme inachevée, mystérieusement interrompue. D’autres

énigmes entourent Manegold : si l'on adopte une vue unitaire de sa biographie, le chevalier alsacien Burgard de Gueberschwihr aurait retrouvé la trace du même penseur en Bavière, à la tête d'un chapitre de chanoines, et l'aurait ramené au pied des Vosges en le chargeant d'y fonder un lieu de réconciliation pour les deux partis que déchirait encore cette querelle. Ainsi naquit le monastère double ${ }^{7}$ de Marbach dans le vignoble haut-rhinois ; ainsi s'épanouit une renaissance alsacienne avec le Codex Guta-Sintram conçu par une moniale et illustré par un moine en préfigurant le Hortus deliciarum du mont Sainte-Odile ; ainsi disparut aussi vers 1103 Manegold de Lautenbach, probablement lors d'une ultime offensive impériale.

Les vingt-neuf premiers chapitres du Livre à Gebhard voient se côtoyer les références tactiques et le monde animal, avec ses bergers ou porchers de qualité variable, ainsi que les pestes et les soins qui se partagent l'être intérieur ; Manegold a surtout mobilisé " la clé du savoir " au chapitre VI avant d'évoquer les " moissons de fidèles " issues du " germe du Verbe " au chapitre X, retraçant les voyages apostoliques de Grégoire VII. Dès lors, il salue en lui un "serviteur des serviteurs de Dieu ", opposant "le témoignage et le goût de la conscience " aux " loups et renards ", aux " impudiques hennissements ", à l'épidémie de simonie comme de nicolaïsme ${ }^{8}$ qui dégrade les ministères de l'Eglise (chapitres XI-XIX). Avant de reprendre une distinction familière à Grégoire VII, abondamment cité, entre "loups " et " pasteurs ", Manegold en introduit une plus révolutionnaire, entre le titre et la qualité d'un dirigeant ; cette intuition permettait de résoudre d'une manière novatrice, retentissante et durable des problèmes linguistiques aussi bien que politiques. Pour Manegold comme pour le pape réformateur, le " glaive spirituel " ${ }^{9}$ est au service de la communion ou, d'après l'expression du pape ${ }^{10}$ Grégoire $1^{\mathrm{er}}$, $\mathrm{du}$ " sourire qui doit faire rayonner le réconfort sur la république de l'univers" (chapitres XX-XXIX).

$\mathrm{Si}$ " sa parole valait une épée " ${ }^{11}$, rappelons avec R. Ziomkowski que chez Manegold prime le "souci de l'âme " ${ }^{12}$ aux accents socratiques. Sans doute cette préoccupation lui fit-elle percevoir, à partir de l'élection de Hildebrand au Saint-Siège sous le nom de Grégoire VII, une équivalence subversive à la fin du chapitre XXIX : " de même qu'évêque, prêtre et diacre ne sont pas des noms dus au mérite, mais qualifient des fonctions, de même roi, comte et duc ne sont pas des noms de nature, mais désignations de fonction ou de dignité ". En tout cas et au-delà des codes médiévaux, le chapitre XXX nous offre la quintessence d'une culture encyclopédique en articulant à la visée d'une radicale démonstration les registres les plus variés : la convergence de l'expérience rustique et de la connaissance historique autant qu'exégétique, l'acuité stylistique et critique, le jeu de l'éloquence pédagogique et de la structure dynamique, tout portait l'aventurier Manegold à réaliser des inventaires pour en élever énergiquement l'inventive synthèse aux dimensions d'une souveraineté populaire ou d'un contrat social... C'était bien de la dynamite, à en juger par le silence qui enveloppa son œuvre jusqu'à nos jours comme par la charge de cette lettre ouverte : une lettre à décacheter et déchiffrer encore, pour aller avec Manegold l'inventurier en avant et pour inventer ensemble $d u$ nouveau. 


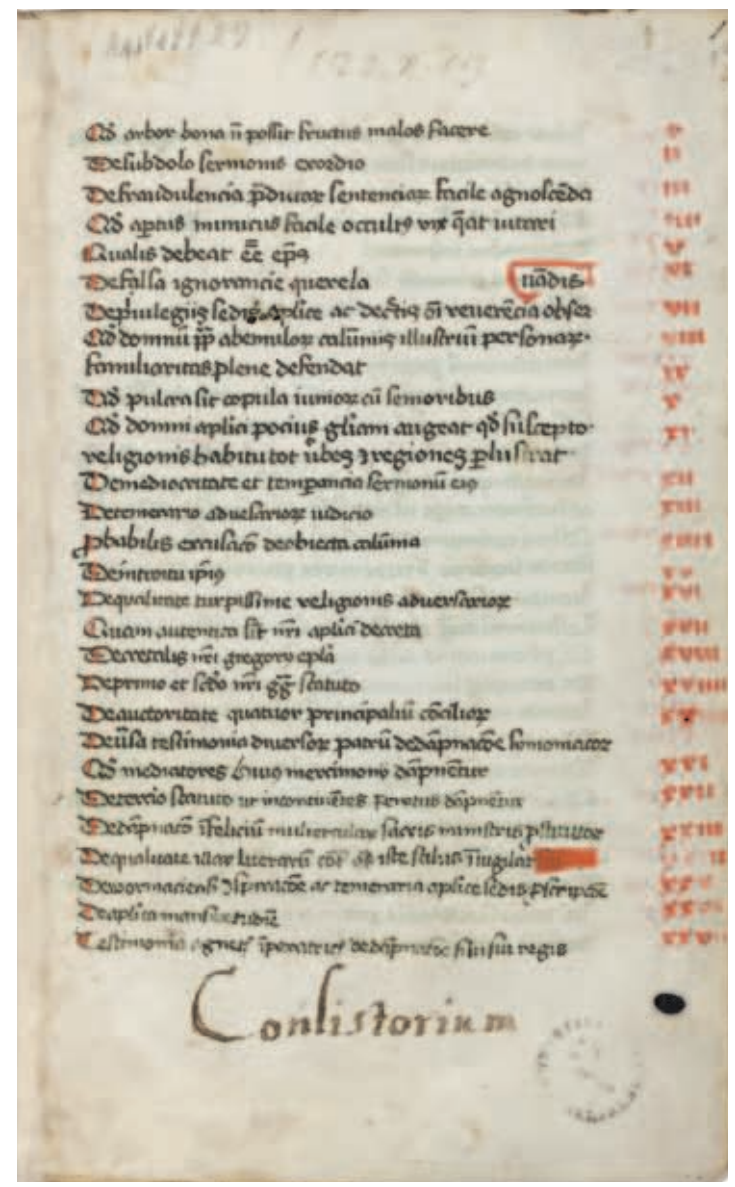

Première page de texte (table des matières) du Livre à Gebhard (coll. Badische Landesbibliothek Karlsruhe)

\section{Chapitre XXX du Livre à Gebhard de Manegold de Lautenbach. Que " roi " n'est pas un nom de nature, mais la désignation d'une fonction.}

Comme la dignité et la puissance royales dépassent tous les pouvoirs de ce monde, de même, pour remplir cette mission, il ne faut pas établir les plus blâmables ou les plus infâmes qui soient, mais celui qui, comme en rang et dignité, surpasse les autres également en goût pour l'essentiel ${ }^{13}$, en justice et piété. Il est donc obligatoire que celui qui doit assumer le soin de tous et gouverner tous les hommes resplendisse, par rapport aux autres, d'une plus grande grâce émanant de ses mérites et s'applique passionnément à remplir la mission qu'il s'est vu confier en la réglant sur la plus haute balance d'équité. En effet, le peuple ne l'élève pas au-dessus de lui en vue de lui accorder la libre faculté d'exercer la tyrannie contre lui, mais pour qu'il le protège contre la tyrannie et la malhonnêteté d'autrui. Cependant, si celui qui est élu ${ }^{14}$ pour refréner les actions dépravées et protéger l'honnêteté a commencé de caresser dans son sein la dépravation, d'écraser les gens de bien et d'exercer lui-même avec le plus de cruauté contre ses sujets la tyrannie qu'il aurait dû bannir, n'apparaît-il pas en toute transparence qu'il tombe à bon droit de la dignité qui lui a été accordée et que le peuple se trouve libéré de la domination et de la sujétion qu'il lui a imposées, puisqu'il est certain qu'il a en premier rompu le pacte au nom duquel il fut établi ? Et personne ne pourra clairement inculper, avec justice et raison, ces gens-là de perfidie puisqu'il est également certain que c'est lui qui en premier a manqué à la loyauté ${ }^{15}$.

En trouvant un exemple parmi des réalités triviales, si quelqu'un confiait à un autre, pour un juste salaire, ses porcs à paître, et qu'ensuite il apprenne que celui-ci 
ne les fait pas paître, mais les vole, les immole et les fait disparaître, est-ce qu'alors, en confisquant même le salaire promis, il ne l'empêcherait pas avec des blâmes de faire paître ses porcs ${ }^{16}$ ? S'il est garanti, dis-je, dans des réalités triviales que même comme porcher ne peut être embauché celui qui s'applique passionnément non à faire paître les porcs, mais à les faire disparaître, quiconque s'efforce non de guider les hommes, mais de les pousser aux errements, est coupé d'autant plus dignement, par une raison juste et acceptable, de toute la puissance et la dignité qu'il assume auprès des hommes que la condition humaine est étrangère à l'espèce porcine. Qu'y a-t-il d'étonnant si ces principes sont garantis sous la religion chrétienne alors que les Romains de l'Antiquité, évidemment à l'époque où vivaient les figures héroïques de Collatin et de Brutus, ne supportant plus l'arrogance du roi Tarquin, pour prix de l'outrage infligé non par lui, mais par son fils, à la noble dame Lucrèce, l'expulsaient au même titre que son fils de sa patrie comme de son royaume et, de manière que personne ne tirât son insolence d'un pouvoir à durée indéterminée, se créaient des magistratures annuelles à travers deux consuls travaillant ensemble désormais ${ }^{17}$ ?

C'est une chose de régner, c'en est une autre d'exercer la tyrannie pendant son règne. De même qu'il faut aux empereurs et rois, pour garder l'œil sur le gouvernail du règne, faire preuve de loyauté comme de respect, de même assurément avec une raison aussi solide, s'ils se sont tout à coup mis à exercer la tyrannie, il leur est impossible, sans porter atteinte à la loyauté ou préjudice à la piété, de sacrifier une forme de fidélité ou de respect : "Car dans le plus vaste pouvoir", dit l'historien ${ }^{18}$, "réside la liberté la plus limitée ". D'où la déclaration d'Augustin contre Novatus ${ }^{19}[. .$. Puisque donc même un léger faux pas ${ }^{20}$ commis par n'importe quelle personne haut placée, comme dit Augustin, est un crime grave, faisons connaître d'autres agissements de votre cher Henri pour montrer à tous ceux qui veulent ouvrir l'œil avec quelle justice il a été déposé ${ }^{21}$, et combien il mérite qu'on lui déverse des malédictions. Donc, pour ne rien dire sur ses adultères innombrables, ses multiples incestes, ses viols, ses nombreuses profanations d'églises par le feu, sur le meurtre de plusieurs milliers d'innocents, sur tout ce dont personne n'ignore la sanction de par les lois divines et humaines, pour passer aussi sous silence le type de faux pas dont la mention souille tout être humain, même s'il le prend simplement, au-delà de ses propres immondices, et pour aller au-delà, dis-je, faisons connaître, à propos de la seule fange sodomique dont il a déjà flétri bien des hommes, ce qu'estiment les règlements sacrés et ce que jugent les divines autorités.

Et de fait le concile ${ }^{22}$ d'Ancyre réuni avant celui de Nicée, mais ensuite confirmé par celui de Chalcédoine, par conséquent jusqu'à la fin du monde compté parmi les règles universelles, énonce là-dessus l'avis suivant au canon XVI : " A ceux qui auraient mené une existence déraisonnable et infligé aux autres la repoussante lèpre d'une faute contraire à toute justice, la sainte assemblée a donné ce conseil : qu'ils disent leurs prières au milieu de ceux qui sont éprouvés par l'esprit impur $"{ }^{23}$. Puisque donc votre cher Henri a complètement flétri une masse de gens par cette impureté, comme le déplorent partout ceux-là mêmes qui ont toléré cet outrage, comment conviendrait-il que régnât sur des chrétiens celui à qui par aucune raison il n'est permis de prier avec les membres du Christ ? De quelle manière conduit-il et guide-t-il l'Eglise du Christ, lui que la même Eglise a compté parmi les possédés et associé aux fous furieux, aux aliénés ? Voyezvous, ô malheureux, voyez-vous, infortunés, ou plutôt vous qui êtes plus misérables que les plus misérables, combien par vous-mêmes vous vous rendez méconnaissables, combien vous vous montrez méprisables, effroyables, voire passibles de malédiction, vous qui vous battez pour que règne sur vous un roi qui a pactisé avec les possédés de l'esprit impur ? En réalité, vous ne sauriez le faire si vous n'étiez vous-mêmes hantés également par l'esprit impur, vous-mêmes dignes qu'un tel personnage vous domine. Vraiment, nul chien ne s'accouple qu'à une femelle de son espèce : à coup sûr, si quelqu'un voyait son chien copuler avec un autre contre nature, il ne lui laisserait pas un jour à vivre. Vous vous montrez donc inférieurs aux chiens, vous qui dans votre folie furieuse vous battez pour placer à votre tête celui qui est plus à maudire que des chiens.

"Mais, disent-ils ${ }^{24}$, personne ne doit être déposé pour punition de ses faux pas : les actes commis ne doivent être reprochés à quiconque ". D'où vient donc, je vous le demande, qu'on lit que les rois mentionnés supra ${ }^{25}$ ont été renversés et les chefs d'innombrables Eglises déposés, sinon de leurs fautes ? Si donc personne, comme ils disent, ne doit être coupé pour prix de ses fautes de la dignité une fois accordée, qu'est-ce qui 
explique, de manière à remonter plus en profondeur vers l'exemple porteur d'autorité là-dessus, que le prototype des créatures ${ }^{26}$ se fait, à l'issue de son faux pas, expulser à l'extérieur des délices du paradis et couper de toute la puissance accordée à l'intérieur ? D'où vient donc que le grand Lucifer qui jaillissait de bon matin $^{27}$, qui montre le premier chemin suivi par les mains divines ${ }^{28}$, que n'ont pas dépassé les cèdres dans le paradis de Dieu, dont les sapins n'ont pas égalé la cime, avec les frondaisons duquel les platanes n'ont pas rivalisé, à la beauté duquel toutes les essences du paradis restent inférieures, qui éclata de beauté dans les feuillages touffus et denses ${ }^{29}$, qui fut le sceau de l'absolue ressemblance, plein de goût pour l'essentiel et parfait relais pour la splendeur de Dieu parmi les délices de Son paradis, dont toute pierre précieuse tissa le manteau, dont l'or fut l'instrument sonnant de splendeur ${ }^{30}$ - d'où vient, dis-je, que ce personnage, largement préféré à tous les chœurs d'en haut de par de célestes ornements remarquables, fut renversé de toute la profondeur du ciel, si personne, comme le proclame le délire bachique de ces individus, ne doit se voir, quelle que soit l'immensité de ses méfaits, coupé d'une dignité une fois accordée?

\section{Présentation et traduction du latin par Martine Hiebel}

\section{Notes}

1 - Drame inédit, cf. Sainte-Beuve, Derniers portraits littéraires, 1852

2 - Caiazzo Irene, Manegold, "modernorum magister magistrorum ", in Arts du langage et théologie aux confins des XI et XII' siècles, sous la direction d'Irène Rosier-Catach, Brepols, 2011, p. 317-347

3 - Les Monumenta Germaniae Historica furent fondés en 1819 par la « Gesellschaft für Deutschlands ältere Geschichtskunde » ou "Société pour l'histoire ancienne de l'Allemagne ". Les deux traités polémiques de Manegold figurent dans la section Libri de lite imperatorum et pontificum ou " Livres sur la querelle des empereurs et des papes".

4 - En Italie du Nord, lieu de la pénitence publique hypocritement acceptée par Henri IV en 1077.

5 - Ziomkowski Robert, Manegold of Lautenbach - Liber contra Wolfelmum, 2002, p. 108-111

6 - Clerc chargé d'une direction pédagogique.

7 - Monastère où moniales et moines vivent dans deux espaces distincts, mais contigus et placés sous une seule autorité spirituelle.

8 - On appelle simonie le trafic des fonctions ecclésiales, et nicolaïsme le refus du célibat sacerdotal.

9- Saint Paul, Lettre aux Hébreux, 4, 12-14 : « Car vivante est la parole de Dieu, agissante et plus perçante que tout glaive à deux lames [... Sur toute créature - jusqu'à la gorge - est clairvoyante la parole qui tient responsable notre parole ".
10- Lettre XIII de Grégoire le Grand, 31. Les références historiques et scripturaires apparaissent pour la plupart dans l'édition des MGH.

11 - Ritter Yves, in Recherches médiévales, $\mathrm{n}^{\circ}$ 6-7, 1984 : tel est le titre de l'article.

12 -Ziomkowski Robert, op. cit., p. 14 : to the care of souls.

13- On trouvera moins insolite cette transposition de sapientia en la rapprochant de la saveur que ce terme implique étymologiquement et de ses nombreuses occurrences chez Manegold.

14-Remarquer l'emploi de ce présent duratif dans l'évocation de l'élection : humaine ou divine, elle est un choix qui revêt pour Manegold le caractère de l'absolu.

15- Ce paragraphe semble connoté par les pactes féodaux en général et, en particulier, par les accords préalables aux Serments de Strasbourg $(842)$ : « Si jamais, ce qu’à Dieu ne plaise, je violais le serment que j'aurai prêté à mon frère, je vous délie tous de toute soumission envers moi, et de la foi que vous m'avez jurée " (chapitre III de l'Histoire des dissensions des fils de Louis le Débonnaire rédigée en latin par Nithart et traduite par F. Guizot).

16- La prophétie d'Ezéchiel contre les mauvais pasteurs et bergers, au chapitre 34 , se reconnaît dans ce truculent décalque.

17- En un raccourci lumineux, à travers un saisissant emploi de l'imparfait duratif et du réfléchi direct, Manegold vient de retracer l'avènement de la toute première république, à Rome en 509 avant Jésus-Christ. Cf. Histoire romaine de Tite-Live, livre II, chapitre 1.

18- Salluste, Conjuration de Catilina, 51

19-Augustin, Contra Novatianum liber supposititius, in Opera III, 2. Cette référence de $\mathrm{K}$. Francke est difficile à confirmer.

20-En latin, une étymologie possible et de nombreux termes de sens voisin autorisent la traduction de peccatum par faux pas.

21-Voir la présentation : se disputant l'autorité de l'investiture ecclésiale, Henri IV et Grégoire VII se sont plus d'une fois destitués.

22- Allusion aux conciles d'Ancyre, de Nicée et de Chalcédoine tenus au $4^{\mathrm{e}}$ siècle et au $5^{\mathrm{e}}$.

23-C'est assez pour suggérer la gravité de l'excommunication infligée par le pape, même sur des motifs plus moraux que légaux.

24- Pensées attribuées à Wenricus comme à l'ensemble du parti impérial par ruse polémique.

25- Le chapitre XXIX vient d'énumérer en les précisant les nombreux cas légitimes de déposition impériale ou royale relevés dans l'ère chrétienne.

26- « Prototype des créatures " voudrait traduire le terme transcrit du grec par Manegold, mis dans la bouche de Salomon en Sg 7, 1 quand il se dit fils d'Adam, et signifiant " façonné en premier ". Remarquer le présent choisi pour cette évocation.

27- Isaïe 14, 12 : c'est le roi de Babylone que vise le prophète. Noter que l'imparfait duratif décrit, conformément à la Vulgate, l'être supérieur déchu qui traverse tous les mythes familiers aux peuples contemporains et voisins d'Isaïe.

28- Job 40, 14 ou 19 : il s'agit de Béhémoth, figure des forces hostiles que Dieu maîtrise autant que la Sagesse ici parodiée, sans doute pour dénoncer Satan ou «l'esprit impur » dont la possession est récurrente en ce chapitre de Manegold.

29-Ezéchiel 31, 8-9 : Manegold insère avec aisance et fidélité ces versets visant la puissance de l'Egypte.

30-Ezéchiel 28, 12-13 : le " maître des maîtres modernes " suit pas à pas la Vulgate en adaptant la prophétie contre le prince de Tyr à son propre combat. La dernière phrase de ce chapitre y reliera d'ailleurs le suivant, qui développera " la condamnation des conspirateurs ». 